\title{
Online Consumer Inspection on Using E-shopping Service of E-commerce
}

\author{
N. Karthikeyan, S. Selva Brunda
}

\begin{abstract}
The Internet has become the primary source of information for a large number of consumers and it enables consumers to share their opinions and experiences concerning goods and services. Online consumer reviews provide information and recommendations for prospective buyers and are useful for decision-making on purchases. The purpose of this study is to assess the impact of on-line consumer reviews on a decision of using e-commerce services. In this study, we tend to conduct a laboratory experiment to analyze product review helpfulness likewise as its corresponding antecedents from the product review feature perspective (i.e., source- and contentbased review features). Findings from the study are threefold. First, the results of the information analysis support the theoretical conceptualization of product review helpfulness as a formative construct. Second, the results support the notion that the source- and content-based review options have direct impact on product review helpfulness. Customers perceive customerwritten product reviews as additional useful than those written by experts; they also perceive a concrete review as additional helpful than an abstract review. Third, we discover an interaction effect of the source- and content-based options of product reviews on review helpfulness. A customer-written product review with a low level of content abstractness yields the highest perceived review helpfulness.
\end{abstract}

Keywords--- Online, Product, Features, Reviews, Helpfulness, Seen, Consumers, Source, Content, Purchases, Internet.

\section{INTRODUCTION}

Internet has rapidly grown during the past decade and its modified consumer behavior in searching pattern from a conventional to digital market as e-commerce. Because the use of the web has spread rapidly, consumers are shifted from a passive to active and informed consumers. Electronic media, like online discussion forum, electronic bulletin board systems, and news teams, are vital sources of informational influence facilitating information exchange among consumers. Web-based technologies have created various opportunities for electronic word-of-mouth (eWOM) communication. With the assistance of web, consumers will notice several information they have concerning merchandise and services that provided by alternative shoppers or to share their opinions and experiences of using goods and services with multitude of alternative consumers. The potential consumers trust online reviews posted by unknown consumers more than they trust traditional media, just like they listen to a private recommendation from friends or family. This development of online orientation impacts retailers as this simply accessible information could greatly affect the consumer behavior on their consumption decision. [1]

\footnotetext{
Manuscript received September 16, 2019.

N. Karthikeyan, Assistant Professor, Department of Computer Science, NGM College, Pollachi, T.N, India. (e-mail: karthikmgm70@gmail.com

Dr.S. Selva Brunda, Professor, Department of Computer Science, Cheran College of Engineering, Karur T.N, India. (e-mail: brindhaselva@yahoo.com)
}

Research has shown that need for social interaction, need for economic incentives, concern for alternative consumers and the potential to enhance their own self-worth are the primary factors that motivate consumers to read-and-write online reviews that are useful on decision-making. in addition, user-generated content (UGC) within the form of online consumer reviews was found to considerably influence consumer purchasing decisions. The name of a product, service or company is not any longer defined by what they report or however they assert they signify. Instead, they're progressively outlined by the shared opinions and experiences of social connected consumers. [2]

A lot of analysis has been done in field of online reviews. However, in current analysis of online reviews, several are involved concerning the impact of on-line reviews on product sales also because the effectiveness of online reviews. The most objective of this study is to assess the impact of the web shopper reviews on consumer decision of using e-commerce services. The study also contributes to the data of marketers by providing insight into consumers' behavior, which may probably be employed by marketers to develop a better strategy of marketing.

\section{LITERATURE SURVEY}

\section{Consumer Buying Decision}

Theories and concepts of online consumer reviews that are utilized in analysis conducted adopting and modifying the theories and ideas derived from the concept which can help explain the various factors of online consumer reviews that have influence on the decision to use the consumer. Online consumer reviews are part of the data that users produce websites that have bought the product targets. Online consumer reviews contain information and proposals on product from a consumer perspective. Some dimensions of on-line shopper reviews were utilized in this study are that the quality argument, supply credibility, timeliness, valence and volume, these dimensions will influence shopper selections. During this case the ideas and theories utilized in this study concerning the definition and indicators of online consumer reviews.[3]

Online consumer Reviews

Online consumer reviews are user-generated content that contain any positive, neutral, and negative comments or reviews of product or services supported customer's experiences. This consumer-created information is useful for decision-making on purchase as a result of it provides customers with indirect experiences. Online consumer reviews are a lot of being relied upon by customers as a low value means of creating more up on getting selections. 
Within the online shopping environment, online consumer reviews play a task of recommendations and spoken. It's been shown that they will be used to reduce consumers' perceived risk of on-line searching and stimulate their purchase intention by providing useful product information. An online consumer reviews as a route for social influence plays 2 roles, as informant and recommender. As an informant role, the online consumer reviews deliver extra user-oriented info, as a recommender role, it provides a positive or negative signal of the product its popularity. [4]

Due to its information asymmetry setting, the web consumer reviews are very important within the ecommerce context. This due the actual fact that the web distributor features a lot more information regarding the product than the consumer has. Compared to traditional shopping, online consumers can't use all of their senses (e.g. touching, smelling) to evaluate the product when they are buying online. This forces shopper to form a buying decision supported the data the distributor provides on his web site or elsewhere. Online consumer reviews offer these information-seeking customers with indirect product and services experiences. Since the fast and still progressively adoption of buying product or services online, online consumer reviews became a lot of $\}$ more necessary. This results in our following hypothesis "Online shopper Reviews features a vital partial and stimulant impact on the consumer decision of using e-commerce services". [5]

\section{METHODOLOGY \& RESULTS}

The basic behavioral event is that the most information element for user behavior analysis. It may be expressed as a triple $\mathrm{BBE}=$. Where: $\mathrm{BBE}$ represents the basic behavior event collected by the browser plug-in, Name indicates the name of the behavior event, Timestamp indicates the timestamp of the basic event, and the url indicates that the behavior event is specific to that page, and the item is optional. [8].

Analysis of the behavioral event set; analysis of the event information set (ABES) is that the basic action event that's processed to create information that facilitates user behavior analysis, analysis of the event information set is outlined as follows: Where: A_1 to aim is the user action event, and also the analysis event information set is that the basis for user behavior analysis. These events include: page dwell time, mouse clicks, page re-visits and also the range of slider movements [10]. The user's interest indicates the degree to that the user is fascinated by a page with certain characteristics. So as to differentiate the user's interest in several web pages, you need to calculate a value for each user's page, that is that the degree of interest. [12]. this text believes that if the user visits an internet page, it should be interested in it [13].

The user behavior model stores the degree of interest of the user to the page predicted by the set of complex events of the user's behavior. The user behavior model may be expressed as. Where: UserlD is used to identify the user; ABES is that the set of complex events of the user's behavior, these events include: page dwell time, mouse

\section{The User Behavior Model for Browsing Web}

clicks, page re-visit times and also the range of slider movements. Interest indicates the user's interest within the page. In this paper, the M5 model tree is used to analyze the information| of users' browsing data [5].

\section{Aggregate ranking algorithm}

In this algorithm we combine the three techniques.

a) Frequency-based method

b) Correlation-based method, and

c) Hybrid method

\section{a. Frequency based Method}

Frequency-based method is the method which is used in our aggregate ranking algorithm, in which it gives the features according to term frequency of the product .This method takes only the frequency of the term and which will impact on the customer opinions on the particular product, it helps in rating the product. There are some usual features of the product will appear frequently those are consider as the important features.

\section{b. Correlation- based Method}

Correlation-based method, which measures the correlation between the reviews on particular products and the final rankings. It ranks the aspects based on the number of cases when such two kinds of opinions are consistent Correlation based method ranks the aspects by simply counting the consistent cases between reviews on particular products and the final rankings. It ignores to model the uncertainty in the generation of overall ratings, and thus cannot achieve satisfactory performance.

\section{c. Hybrid Method}

Hybrid method, that captures both aspect frequency and the correlation the hybrid method simply aggregates the results from the frequency-based and correlation-based methods, and cannot boost the performance effectively.

\section{Proposed System}

We mainly study on the feature extraction issue from user behavior logs, and then try to predict purchase behaviors in online shopping sites utilizing the neural network classification algorithm in data mining.

\section{A. User Click Behavior Analysis}

In order to get useful and effective classification features, we need to thoroughly study the relationships between users' click behavior and their purchase behavior by investigating some statistics of the information. All the behaviors done by constant user to the same item constitute a session, identified by user-item pair.

First, we examine a number of properties that the dataset share and observe however users behave once they decide to get something. We discover that users tend to have much more click behaviors when they need to buy some item than when they show no interest within the item or they're not satisfactory to the item. In $73.3 \%$ of purchase sessions user's total click behavior number is over 5 times, except for non-purchase sessions, only $7.7 \%$ of them have constant

Published By:

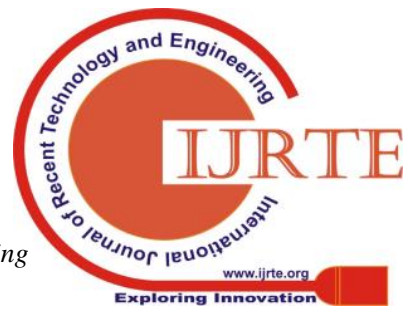


Click behavior range. It implies that it'd be effective to predict users' purchase by making use of their click behavior numbers.

The purchase probability distribution of the quantity of clicks for all user-item sessions. With the rise of the quantity of clicks, the purchase probability is additionally raised. Before creating his/her purchase, a user can click the item persistently. In Tianchi knowledge set, a user would click nine times in average before purchase. This clicking history actually records however users act with the system and perform their purchases, whereas previous cooperative filtering algorithm fails to exploit it.

We found that if an individual is willing to buy something on an e-commerce site he's likely to spend more time on it. So the total number of clicks and the entire time span of a session may be used as indications of a user's general perspective towards online shopping. we also found some options about users include how usually the user would purchase, what number times the user would click the item in average once they want to purchase, and so on. These features aren't the behavior directly happened between the users and item, however will show the standard purchase habits and patterns of the user. And that we will get them from the statistics information of users' behavior log.

The features about items themselves are similar. we don't have the data from original dataset about items, such as item name, price, sales, however we will also get the useful content from the statistics information about the item, like how many times the item has been clicked, else into the shopping cart and purchased. We observe that the purchase probability changes a lot over time. so as to find the relationships between clicking time and get behavior, we tend to counted the quantity of purchase behaviors happened in several time span. As a consequence, the purchase behavior is extremely correlative with user's last visit time, the willingness of purchase decays fast when time grows. This is reasonable consistent with our experience. Individuals tend to buy items that they visited in recent time instead of a long time ago. To exploit this data, we tend to extract hour as features from the time when user click the item.

\section{B. Feature Extraction}

Based on the observation above and previous works, we build our features from the following aspects:

1. For the users, we design features that reflect his/her purchase habit, preference and pattern, such as adding to the shopping cart before purchase, or click more than 9 times before purchase;

2. For the items, features are these which describe the inherent property of each item, like the number of visitors, number of click behaviors, and number of purchase behavior happened;

3. For the sessions, we focus on user recent behaviors on this item, which represents his/her intention to buy or notsuch as how many times the user has browsed the item, how many times the user have added the item into his favorites and shopping car;

4. For the time aspect, we designed the features like the count of clicks happened in each hour, the first time (include the month, day and hour) and the last time the user clicked the item, the count of the different time period user clicked the item, average click number in each time period, and the average interval between two adjacent time periods;

5. For the categories, features capture the interactions between users, items and the corresponding item categories. For example, the feature of the total time that a user has spent on a certain item category can reveal the user's preference on this item category. The different click numbers between the given item and other items in the same category can tell why the user chooses to buy this item not others similar.

What's more, all of these features can be extended to different time span to get more features for purchase prediction.

In classification problems, both Binary and Real-valued feature models have been widely used. In Binary-valued feature model, the feature value is either 1 or 0 indicating whether or not a particular phenomenon occurs in the session. In Real-valued feature model, the feature usually refers to the frequency which is defined as the number of times that a particular click behavior appears in the session. To apply these features for classification, we convert these numerical features into logical features and build a Bayesian classification rule.

\section{C) Ranking the Customer Reviews}

We propose a product aspect ranking framework to automatically filter the important aspect of products from lots of consumer reviews. Developing probabilistic aspect ranking algorithm to clear the importance of many more aspect by simultaneously exploiting aspect frequency and the impact of consumer opinions on the product .Demonstrate the capabilities of aspect ranking in real world applications. Performance improvement are obtained on the applications of document level sentiment classification and extractive review summarize by making use of aspect ranking

\section{D) Computational Model}

In product aspect ranking framework consisting of three main components, Aspect identification, Sentiment analysis, Aspect ranking.

\section{Aspect Identification}

We identify the aspects by kept the frequent noun terms in the consumer reviews. Previous studies aspects are nouns or noun phrases. For identify aspect existing aspect identification approach. In existing approach first identify noun and noun phrases in the document. The counted occurrence frequencies of noun and noun phrases and only frequent one are kept as aspects. Limitation is that the identified aspects usually contain noise in proposed system split free text reviews into sentence, using Stanford parser parse each sentence. The frequent noun phrases are extracted from parsing trees as candidate aspects. These usually contain noise. Assist identify aspects from the candidate using pros and cons reviews.

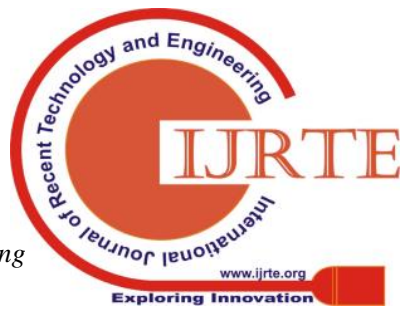


In this use synonym clusters for identify unique aspects from reviews.

\section{Sentiment Analysis}

The sentiment classification is done using naïve Bayes model classifier. Pros and cons reviews categorized positive and negative opinion on the aspects. These reviews used as training samples for learning sentiment classifier, which is used for determine consumer opinion on the specific aspect in free text reviews. First collect the sentiment terms from pros and cons reviews. Then the trained classifier using these sentiment terms and this trained classifier is used to classify the aspect in free text reviews.

\section{Aspect Ranking}

The consumer's opinion on each aspect is found. A Probabilistic aspect ranking algorithm is used for rank the aspects according to account aspect frequency and the influence of consumer's opinion given to each aspect over their overall opinion. Important aspects have some characteristics: a] Important aspect is frequently commented in reviews. b] Customer opinions on frequent aspect is greatly influence their overall opinions of the product. The aggregation of the opinion is given to specific aspect in the reviews that is overall opinion, and various aspects use different contributions in the aggregation.

\section{Artificial Neural Networks (ANN)}

ANNs constitute an information-processing paradigm that is inspired by biological nervous systems [17]. The key element is the novel structure of the paradigm. It is made up by a large number of highly interconnected processing neurons working in unison to solve specific problems. In this study, the back propagation neural network ANNs was employed.

\section{Back-propagation Neural Network}

Back-propagation is a commonly used learning algorithm in ANN applications. It uses the gradient descent algorithm to determine the weights in the network. Associate ANN consists of 3 or more layers: an input layer, a hidden layer(s), and an output layer. The input layer contains input nodes (neurons), i.e. the input variables for the network. The output layer contains the desired output of the system, and the hidden layer usually contains a series of nodes related to a transfer function. Each layer of the network is linked by weights that need to be determined through a learning algorithm. Sigmoid function could be a commonly used transfer perform, that is adopted as the property that it squashes the variable, which can have a range from - to, to the range 0-1 [8]. At the output layer wherever the network output must be compared with the target output, the target values need to be standardized to the vary of $(0,1)$.

Deep Learning is an important aspect of sentiment analysis. It shows better results that help to gain the efficiency in Sentiment Analysis. The approach based on deep Learning uses a Convolutional Neural Network (CNN) inspired through Deep Learning algorithm. CNN uses word2vec () to get the vector representation of word as associate input. This improves the performance of the network and helps in gaining the potency [16]. It additionally uses parametric rectified linear unit (PReLU), normalization and Dropout technology which is able to increase the accuracy and maintain the generalizability of the model.

A model using Artificial Neural Network for sentiment analysis of client reviews is planned. This technique classified the review into positive, negative and fuzzy tone [18]. The proposed approach combines the benefits of the machine learning techniques and the information retrieval techniques [19].

\section{Result}

Without feature extraction prediction and classification

\section{Performance analysis without feature extraction}
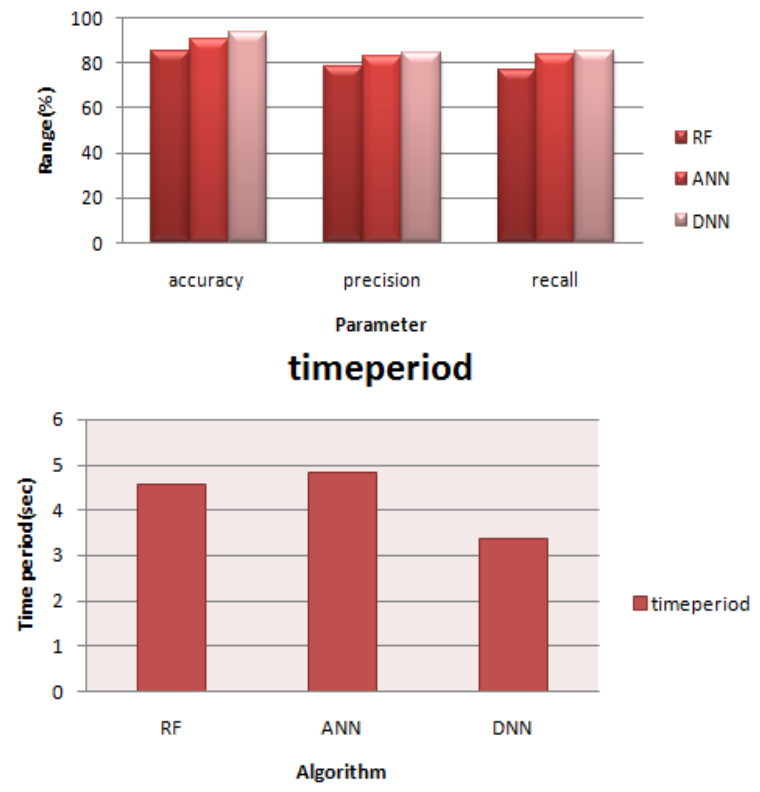

With feature extraction prediction and classification performance analysis with feature extraction
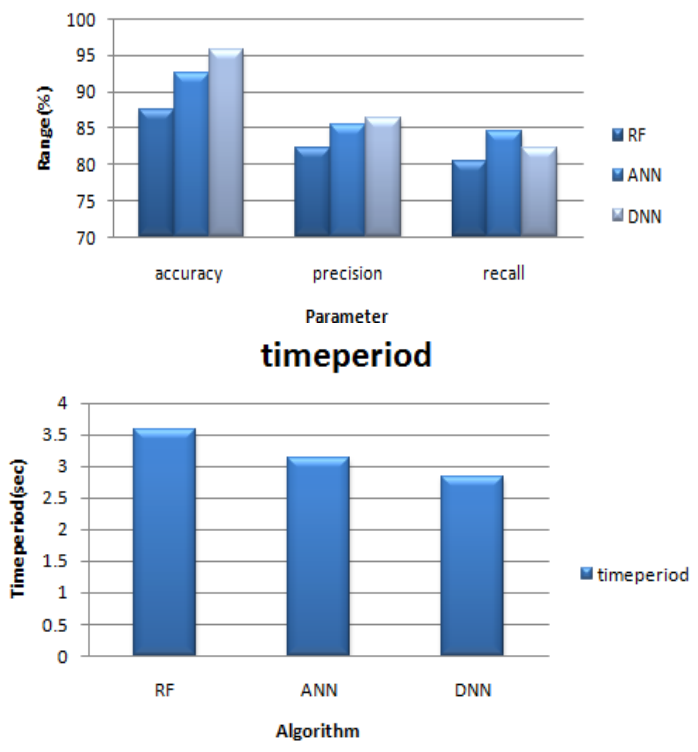

Published By: Blue Eyes Intelligence Engineering 


\section{CONCLUSION}

User behavior analysis is through the way of data mining from a large number of network information mining user behavior patterns. It is a relatively new research field, has a wide range of application prospects, become a hot topic of domestic and foreign scholars.

This paper mainly studies the behavior analysis method of user interest, studies the Web log mining method, and puts forward the calculation degree of user's interest in web pages based on online shopping. The process of constructing user behavior model based on prediction of reviews using the artificial neural network. Finally, we use the collected reviews of the customers and analyzed using the algorithms.

\section{REFERENCES}

1. Jun Zha, Jianxing Yu, Jinhui Tang, Meng Wang Product Aspect Ranking and Its Applications. IEEE TRANSACTIONS ON KNOWLEDGE AND DATA ENGINEERING, VOL. 26, NO. 5, May 2014.

2. Baek, H.; Ahn, J., and Choi, Y. Helpfulness of online consumer reviews: Reader's objectives and review cues. International Journal of Electronic Commerce, 17, 2 (2012).

3. Tarhini A, Arachchilage N A G and Abbasi M S 2015 A critical review of theories and models of technology adoption and acceptance in information system research International Journal of Technology Diffusion (IJTD) 6 (4) 58-77

4. Sparks B A and Browning V 2011 The impact of online reviews on hotel booking intentions and perception of trust Tourism Management 32 (6) 1310-1323

5. Chiu C M, Chang C C, Cheng H L and Fang Y H 2009 Determinants of customer repurchase intention in online shopping Online information review 33 (4) 761-784

6. SoroushVosoughi, Helen Zhou, and Deb Roy. Enhanced customer sentiment classification using contextual information. arXiv preprint arXiv:1605.05195, 2016.

7. Wan Fei, Zhao Xi, Liang, and so on. Research on Search Engine User Behavior Based on Mobile Internet Log. Chinese Journal of Information, 2014, 28 (2): 144-150.

8. Cen Rongwei, Liu Yiqun, Zhang Min, et al. Search Engine User Behavior Analysis Based on Log Mining. Chinese Journal of Information, 2010,24 (3): 49-54.

9. RongGuoting, Luo Yong, Sun Jianjun. Research on Library User's Behavior Based on Log Analysis. Library Journal, 2015(7): 59-63.

10. N. Ghahreman and M. Sameti, "Comparison of M5 model tree and Artificial Neural Network for estimating Potential Evapotranspiration in semi- arid climates," Department of Irrigation and Reclamation Engineering, University of Tehran, Karaj, Iran, March 2014

11. G. Vinodhini and RM. Chandrashekharan, "Sentiment Analysis and Opinion Mining: A Survey", International Journal of Advanced Research in Computer Science and Software Engineering, Volume2, Issue 6, June 2012.

12. DudhatAnkitkumar, "A Survey on Sentiment Analysis and Opinion Mining “, International Journal of Innovative Research in Computer and Communication Engineering Vol. 2, Issue 11, November 2014.

13. ZohrehMadhoushi, Abdul Razak Hamdan and Suhaila Zainudin "Sentiment Analysis Techniques in Recent Works", Science and Information Conference (SAI), 2015. IEEE, 2015.

14. AnkitGoel and ParulGarg (2014), A Study on Consumer Preferences for E Shopping, International Journal of Applied Research in Engineering and Science (IJARES), Vol 1 Issue 1, ISSN 2347-9337.

15. AtherAkhlaq et.al (2014), Online shopping A Global Perspective, Journal of Basic and Applied Scientific Research, Vol 4 (5) pp 153-160, ISSN 2090-4304.

16. Prashantsingh (2014), Consumers Buying Behaviour Towards Online Shopping (A case study of Flipkart.com user's in Lucknow city), Journal of Research In Commerce and Management Vol 3, pp 27-34, ISSN $2277-1166$

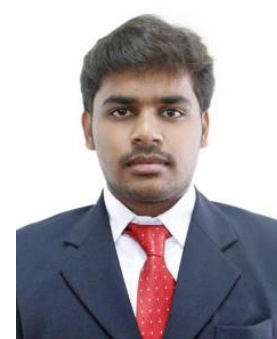

user prediction.

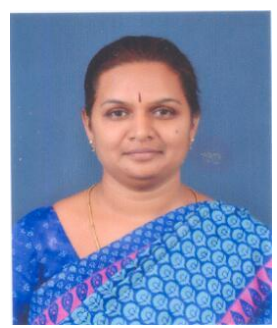

Dr.S. Selva Brunda, Received Ph.D Degree from Mother Teresa University, Kodaikanal, India in 2012. She has been working as Professor \& Head Department of Computer Science and Engineering at Cheran College of Engineering, Karur. Her main area of research interests are data mining, Cloud Computing, Artificial Intelligence\& Game Theory. She received young women scientist award from Dr.Abdul Kalam trust for education for the year 2016. 